\title{
LIVING-DONOR LOBAR LUNG TRANSPLANTATION EXPERIENCE: INTERMEDIATE RESULTS
}

Vaughn A. Starnes

Mark L. Barr

Robbin G. Cohen

Jeffrey A. Hagen

Winfield J. Wells

Monica V. Horn

Felicia A. Schenkel
Objective: Living-donor lobar lung transplantation offers an alternative for patients with a life expectancy of less than a few months. We report on our intermediate results with respect to recipient survival, complications, pulmonary function, and hemodynamic reserve. Methods: Thirty-eight living-donor lobar lung transplants were performed in $\mathbf{2 7}$ adult and $\mathbf{1 0}$ pediatric patients for cystic fibrosis (32), pulmonary hypertension (two), pulmonary fibrosis (one), viral bronchiolitis (one), bronchopulmonary dysplasia (one), and posttransplantation obliterative bronchiolitis (one). Seventy-six donors underwent donor lobectomies. Results: There were 14 deaths among the 37 patients, with an average follow-up of 14 months. Predominant cause of death was infection, consistent with the large percentage of patients with cystic fibrosis in our population. The overall incidence of rejection was 0.07 episodes/patient-month, representing 0.8 episodes/patient. Postoperative pulmonary function testing generally showed a steady improvement that plateaued by postoperative months 9 to 12. Fourteen patients who were followed up for at least 1 year underwent right heart catheterization; pressures and pulmonary vascular resistances were within normal ranges. Bronchiolitis obliterans was definitively diagnosed in three patients. Among the 76 donors, complications in the postoperative period included postpericardiotomy syndrome (three), atrial fibrillation (one), and surgical reexploration (three). Conclusions: We believe that these data support an expanded role for living-donor lobar lung transplantation. Our intermediate data are encouraging with respect to the functional outcome and survival of these critically ill patients, who would have died without this option. (J Thorac Cardiovasc Surg 1996;112:1284-91)
$R^{2}$ ecent improvements in survival and quality of life have made pulmonary transplantation a therapeutic option for adults and children with end-stage lung disease, whether caused by parenchymal or by vascular lesions. ${ }^{1-4}$ As a result of progress in this field, the number of patients waiting for lung transplants has grown, while the number of donors

From the Division of Cardiothoracic Surgery, University of Southern California School of Medicine, Los Angeles, Calif.

Read at the Seventy-sixth Annual Meeting of The American Association for Thoracic Surgery, San Diego, Calif., April 28-May 1, 1996.

Received for publication May 6, 1996; revisions requested June 10, 1996; revisions received July 15, 1996; accepted for publication July 17, 1996.12/6/76551

Address for reprints: Vaughn A. Starnes, MD, Professor and Chief, Division of Cardiothoracic Surgery, School of Medicine, 1510 San Pablo St., Suite 415, Los Angeles, CA 90033-4612.

Copyright (C) 1996 by Mosby-Year Book, Inc.

$0022-5223 / 96 \$ 5.00+0 \quad \mathbf{1 2 / 6 / 7 6 5 5 1}$ has remained relatively unchanged. According to the 1995 United Network for Organ Sharing (UNOS) Registry, ${ }^{5} 1939$ patients are waiting on the recipient list in the United States for lung transplantation, with the expectation that because of the donor shortage only 700 to 800 of these patients will receive transplants. The resulting mismatch between the growing number of recipients and the static pool of donors translates into an increasing number of deaths among patients on the waiting list.

The concept of lobar transplantation was first developed as an alternative to whole-lung transplantation in children. ${ }^{6}$ The use of lobar transplantation made cadaveric adult donors available to small adult and pediatric recipients. ${ }^{7,8}$ Living-donor lobar lung transplantation offers an alternative form of treatment for patients who have a decline in physical status with a life expectancy of less than a few months or increasing risk with time of the development of an absolute contraindication to transplan- 
tation. We report on the intermediate results with respect to recipient survival, complications, pulmonary function, and hemodynamic reserve.

\section{Patients and methods}

Recipient selection. From January 1993 to April 1996, 38 bilateral living-donor transplants were performed in 37 patients. Twenty-seven patients were adults, with a mean age of 25 years (range 18 to 36 years), and 10 were children, with a mean age of 13 years (range 9 to 17 years). There were 15 male and 22 female recipients.

Recipients were selected on the basis of deteriorating status and the expectation that a cadaveric donor would not become available in time. Of the 37 patients who underwent transplantation, 22 came from out of state, were transported on an emergency basis, and underwent transplantation within 72 hours of arrival.

Most patients in our group were critically ill, as illustrated by the number of those with marked weight loss, steroid dependence, increasing hypoxia or hypercapnia, increasing antibiotic resistance, deteriorating pulmonary function test values, recurring life-threatening pulmonary events (e.g., hemoptysis), and decreasing ability to perform the activities of daily living (Table I).

The indications for lobar transplantation were cystic fibrosis $(n=32)$, pulmonary hypertension $(n=2)$, pulmonary fibrosis $(n=1)$, viral bronchiolitis $(n=1)$, bronchopulmonary dysplasia $(n=1)$, and posttransplantation obliterative bronchiolitis $(n=1)$. All patients fulfilled the criteria for cadaveric lung transplantation and were listed with UNOS before lobar transplantation. Clinical deterioration without expectation of a cadaveric allograft was used as the criterion for entry into the living-donor lobar transplantation protocol.

Absolute contraindications to lobar transplantation include panresistant flora. Patients must have no significant irreversible end-organ damage other than their pulmonary disease. Creatinine clearance studies are performed to assess renal status, especially for patients who received repeated courses of aminoglycosides. Echocardiographic assessment of left and right heart function is performed. Cardiac catheterization may be performed to determine pulmonary artery pressures. Seroligic testing includes cytomegalovirus; Epstein-Barr virus; hepatitis A, B, and $\mathrm{C}$; toxoplasmosis; and human immunodeficiency virus; results are obtained at the time of evaluation. Bacterial infections, as demonstrated by an increasing white blood cell count, positive culture results, or fever, were treated before transplantation and immunosuppression.

Relative contraindications to lobar transplantation include severe cachexia, pleural adhesions, diabetes, and long-term ventilatory support. A history of major thoracic surgery or pleurodesis is considered on a case-by-case basis. Patients with diabetes are considered if they have no other end-stage organ dysfunction. Patients receiving short-term ventilatory support are considered and undergo panculture to assess for infection before transplantation. Patients with decreased bone density are evaluated and may be placed on new prophylactic regimens.

All patients must have the emotional and educational ability to undertake a major medical event and its se-
Table I. Preoperative data, severity of illness

$\begin{array}{ll}\text { In hospital } & 25 / 38(66 \%) \\ \text { Preoperative steroids } & 19 / 38(50 \%) \\ \text { Marked weight loss (\% predicted) } & 76 \% \\ \text { Hypoxia } & \\ \quad \text { Ventilator dependent, } 1.00 \mathrm{FiO}_{2} & 6 \\ \quad \text { Bipap } & 3 \\ \text { Hypercapnia } & 69(36-160) \\ \mathrm{PCO}_{2}(\mathrm{~mm} \mathrm{Hg}) & 19 \%(8 \%-30 \%) \\ \mathrm{Mean} \mathrm{FEV}_{1}(* \% \text { predicted) } & \end{array}$

$\mathrm{FiO}_{2}$ Inspired oxygen fraction; $\mathrm{PCO}_{2}$, carbon dioxide fraction; $\mathrm{FEV}$, forced expiratory volume in 1 second.

quelae. Ideally, the patient's support structure should be identified before transplantation, because the people who form this structure are crucial throughout all the phases of transplantation.

Donor selection. Initially, as per our original institutional review board protocol at the University of Southern California and Childrens Hospital Los Angeles, parents were the only donors used for living-donor lobar transplantation. This restriction limited the ability to offer this option to patients whose parents were not considered suitable donors because of results of diagnostic testing or age. Living-donor lobar transplantation was subsequently expanded to include all extended relatives and also unrelated donors. The evolution was gradual and in conjunction with the institutional review board.

All potential donors evaluated have the knowledge and desire to pursue the option of donation. Preliminary testing includes $\mathrm{ABO}$ compatibility testing, chest radiography, and spirometry. Extensive psychosocial assessment is performed by the transplant coordinator, social worker, and team psychiatrist. All donors and recipients are interviewed together to assess family dynamics and separately to avoid coercion. Preliminary screening allows a limited number of potential donors to be evaluated more fully, thereby decreasing costs.

Potentially suitable donors undergo medical screening that includes the following: spirometry with arterial blood gas analysis, routine transplant serologic studies, sputum cultures, viral screening, electrocardiography, echocardiography, quantitative ventilation and perfusion scan with differential split and segmental analysis, and high-resolution chest computerized tomography to exclude occult pathology and to make a volumetric assessment of the lobe to be removed.

Seventy-six donors underwent donor lobectomies. They had a mean age of 40 years (range 18 to 55 years), a mean height of $170 \mathrm{~cm}$ (range 155 to $191 \mathrm{~cm}$ ), and a mean weight of $71 \mathrm{~kg}$ (range 50 to $105 \mathrm{~kg}$ ). Seventy-three donors were related to their recipients and three were unrelated.

Operative procedures

Donor lobectomy. In the removal of a healthy lobe for transplantation, the procedure must be conducted to avoid any compromise to the patient. The operation evolved with this premise in mind. The right lower lobe is removed as the graft for the right lung in the recipient and 
the left lower lobe is removed as the left lung graft in the recipient. The right and left lower lobes have become the standard lobes removed. The anatomy of these lobes has permitted their removal with minimal complications in the donor and has provided adequate pulmonary reserve in the recipient. The complete details of the surgical technique have been described elsewhere. ${ }^{7}$ Before incision, the donors undergo preoperative bronchoscopy to assess for any occult disease and to determine bronchial anatomy. This procedure resulted in the diagnosis of an endobronchial lesion in one patient, precluding donation at the time. Prostaglandin $\mathrm{E}_{1}$ is administered intravenously to dilate the pulmonary vascular bed and the dosage is adjusted to maintain a systolic blood pressure of 90 to $100 \mathrm{~mm} \mathrm{Hg}$. Several technical points should be emphasized. Manipulation of the lobe is kept to a minimum, fissures are developed with surgical staplers to avoid air leaks in both donor and recipient, and complete dissection of the bronchus, artery, and vein is performed, with their relationship to the remaining lobes noted before division. After the dissection is completed, heparin $(300 \mathrm{U} / \mathrm{kg}$ ) and methylprednisolone (Solu-Medrol) are given intravenously, followed by the division of the pulmonary artery, vein, and bronchus. This order of division is important to avoid congestion of the lung graft.

Donor lobe preservation. The lobe is removed and taken to a sterile back table. The pulmonary artery and pulmonary vein are alternately perfused with approximately $2 \mathrm{~L}$ modified Euro-Collins solution $\left(4^{\circ} \mathrm{C}\right)$ until uniform blanching of the organ is achieved. The lobe is simultaneously ventilated with room air to remove any areas of atelectasis. The bronchus is atraumatically clamped while the lobe is partially inflated, and the lobe is stored in cold saline solution $\left(4^{\circ} \mathrm{C}\right)$ and transported to the recipient's operating room in an ice-filled cooler. The chest is closed in routine manner, and an epidural catheter is placed for administration of analgesics. The donor is moved to the Thoracic Intensive Care Unit.

Recipient operation. At the same time that the two donor operations are being performed, the recipient operation is started in a third operating room. The recipient operation is conducted through a bilateral thoracosternotomy (clamshell) incision. ${ }^{8}$ Preliminary dissection is carried out to free adhesions that might be present in the apical and diaphragmatic surfaces of the thorax and medially next to the pericardial surfaces. Once this dissection is completed and bleeding is controlled, the patient is placed on cardiopulmonary bypass and the lungs are removed. In patients with cystic fibrosis, the chest is irrigated with tobramycin solution. Cardiopulmonary bypass is employed for two reasons. First, the patients in this study were in such critical condition that single-lung ventilation could not be performed. Second, and most important, having all of the cardiac output driven through one lobe while the other lobe was being implanted would most likely result in reperfusion pulmonary edema. The cardiopulmonary bypass circuit is therefore used to allow simultaneous reperfusion of both lobes in a controlled fashion.

The sequence of the recipient anastomosis is the bronchus, vein, and artery. The bronchial anastomosis is completed with running 4-0 polypropylene suture with minimal telescoping of the donor's lobe bronchus into the recipient's. By limiting the amount of peribronchial dissection and using this technique of anastomosis, we have avoided bronchial complications. The pulmonary vein of the donor is anastomosed to the superior pulmonary vein of the recipient. The artery is anastomosed end-to-end with 5-0 polypropylene suture. At the conclusion of the transplant procedure, transesophageal echocardiography is performed to ensure that there are no technical complications with venous anastomosis. The mean ischemic time of the right lobe is 65.5 minutes (range 32 to 142 minutes); that of the left lobe is 58.7 minutes (range 34 to 112 minutes).

Recipient postoperative management and immunosuppression. Low-dose dopamine and dobutamine are used for cardiovascular support as needed. The recipient is ventilated through a single-lumen tube with increased positive end-expiratory pressure of 5 to $10 \mathrm{~mm} \mathrm{H}_{2} \mathrm{O}$ for approximately 48 to 72 hours. This prolonged ventilatory support is provided in an attempt to decrease atelectasis and maintain maximal expansion of the relatively undersized lobes. In addition, we believe that this precaution may decrease reperfusion injury in this clinical setting, in which the entire cardiac output is flowing through only two lobes. Chest tubes are set to minimal suction during the first 12 to 24 hours. Several patients were difficult to ventilate if the chest tubes were set to the conventional 20 $\mathrm{cm} \mathrm{H}_{2} \mathrm{O}$. This problem is exaggerated when there is a large discrepancy between the sizes of the lobe and the thoracic cavity. After the initial period of nil to minimal suction, increased suction is started on one tube at a time until all tubes are at $10 \mathrm{~cm} \mathrm{H}_{2} \mathrm{O}$ suction. If this is tolerated, the tubes are then sequentially increased to 20 $\mathrm{cm} \mathrm{H}_{2} \mathrm{O}$ suction. The chest drains remain for a longer period ( 2 to 3 weeks) than with conventional cadaveric lung transplantation because of persistent serous drainage, which is probably a result of the size and topographic mismatch of the lobe. A thoracic epidural catheter is placed when the patient is ready for extubation and after results of coagulation studies have returned to normal.

A noninduction immunosuppressive protocol with cyclosporine, azathioprine, and prednisone was used for most patients. Induction therapy with monoclonal or polyclonal antibodies has been avoided because of concerns with infection, especially in view of the large proportion of cystic fibrosis patients in our cohort. In 1995, use of the microemulsion formulation of cyclosporine (Neoral) was instituted because of its better absorption. FK 506 (Prograf) was also used in the three most recent patients. Before operation, the patient is given $2 \mathrm{mg} / \mathrm{kg}$ cyclosporine intravenously or $0.1 \mathrm{mg} / \mathrm{kg}$ FK 506 orally and is given $4 \mathrm{mg} / \mathrm{kg}$ azathioprine intravenously. During operation, $15 \mathrm{mg} / \mathrm{kg}$ methylprednisolone is given after cardiopulmonary bypass is terminated. After operation, 6 $\mathrm{mg} \cdot \mathrm{kg}^{-1} \cdot$ day $^{-1}$ cyclosporine is given and the dose is adjusted to achieve a whole-blood level of approximately 300 to $350 \mathrm{ng} / \mathrm{ml}$. For those patients receiving FK 506, doses of $0.2 \mathrm{mg} \cdot \mathrm{kg}^{-1} \cdot$ day $^{-1}$ are administered and adjusted to achieve levels of 10 to $20 \mathrm{ng} / \mathrm{ml}$. Azathioprine at a dose of $2 \mathrm{mg} / \mathrm{kg} / \mathrm{day}$ is given and adjusted to achieve a white blood cell count of approximately $6000 \mathrm{cells} / \mathrm{mm}^{3}$. Corticosteroids are administered to achieve a prednisone 


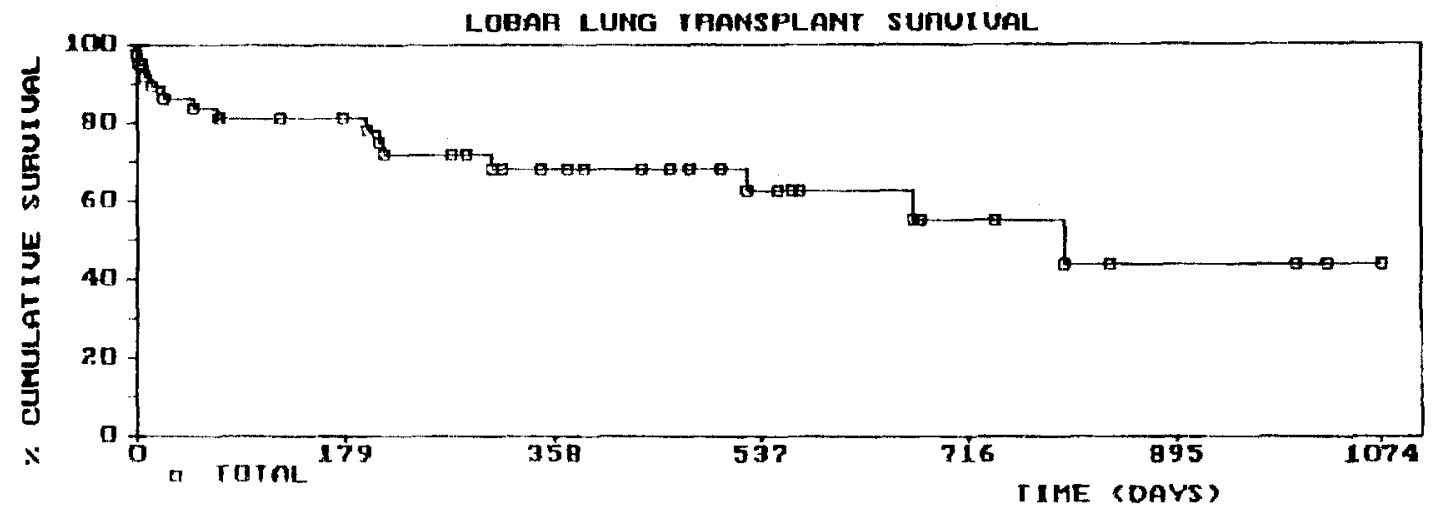

Fig. 1. Kaplan-Meier survival curve after live-donor lobar lung transplantation.

dose of $0.5 \mathrm{mg} / \mathrm{kg} /$ day by postoperative day 30 . This dose is decreased by $0.1 \mathrm{mg} \cdot \mathrm{kg}^{-1} \cdot \mathrm{day}^{-1}$ until the patient has reached a long-term maintenance dose of $0.1 \mathrm{mg}$ to 0.2 $\mathrm{mg} \cdot \mathrm{kg}^{-1} \cdot$ day $^{-1}$.

Pneumocystis carinii prophylaxis consists of trimethoprim-sulfamethoxazole $(320 / 1600 \mathrm{mg})$ given three times a week for the first year. Cytomegalovirus (CMV) prophylaxis is given to all patients with a CMV-positive donor, regardless of recipient preoperative status. Prophylaxis consists of $150 \mathrm{mg} / \mathrm{kg}$ CMV hyperimmune globulin intravenously on postoperative days 1 and 21. Ganciclovir is given intravenously at a dose of $6 \mathrm{mg} / \mathrm{kg} /$ day on postoperative days 14 through day 42 . Acyclovir is given orally at $2400 \mathrm{mg} /$ day thereafter for the first 6 months, as well as to all recipients with CMV-negative donors.

Monitoring for infections and rejections is essentially the same as for cadaveric lung transplant recipients. Transbronchial biopsies are performed only when clinically indicated.

\section{Results}

There were 14 deaths among the 37 patients, with an average follow-up of 14 months (range 1 to 34 months). The 1-year Kaplan-Meier survival was $68 \%$ (Fig. 1). Infection was the predominant cause of death, consistent with the large percentage of patients with cystic fibrosis in our population (Table II). The incidence of infections was 0.13 infections/patient-month, representing 1.6 infections/patient. Most infections resulting in morbidity and mortality were caused by Pseudomonas aeruginosa (Table III).

The overall incidence of rejection was 0.07 episodes/patient-month, representing 0.8 episodes/patient. Of the 30 episodes of rejection seen in the 37 patients, 93\% (28 episodes) were unilateral; only two episodes were bilateral and synchronous. Twenty-nine episodes $(96 \%)$ were mild and classified as
Table II. Causes of death $(n=14)$

\begin{tabular}{ll}
\hline Disseminated Aspergillosis & 2 \\
Sepsis & 2 \\
Pneumonia/sepsis & 4 \\
Obliterative bronchiolitis & 2 \\
Obliterative bronchiolitis/sepsis & 2 \\
Renal failure, cerebral edema & 1 \\
LAD thrombus & 1 \\
\hline
\end{tabular}

$L A D$, Left anterior descending coronary artery.

Table III. Infection

\begin{tabular}{lr}
\hline Pseudomonas pneumonia & 24 \\
Pseudomonas sepsis & 2 \\
Staphylococcus pneumonia & 4 \\
Symptomatic CMV & 6 \\
Aspergillosis & 5 \\
Candida bronchitis/pneumonia & 3 \\
Other & 6 \\
\hline
\end{tabular}

Total incidence 0.13 infections/patient-month, 1.6 infections/patients.

grade A2 according to the International Society for Heart and Lung Transplantation grading system. There was only one rejection episode classified as grade A3.

All episodes responded to an augmentation in steroid dose. Four patients who had more than two episodes of rejection had additional therapy with FK 506 as a replacement for cyclosporine and cyclophosphamide as a replacement for azathioprine, with or without the addition of methotrexate.

The average number of HLA matches was 2.9, with a range of 0 to 6 , and the average number of mismatches was 2.7, also with a range of 0 to 6 . To date there has been no clear pattern in which lobe 
Table IV. Postoperative pulmonary spirometric data

\begin{tabular}{lcc} 
& Mean (\%) & Range (\%) \\
\hline FVC & 72 & $50-103$ \\
FEV $_{1}$ & 73 & $53-95$ \\
DLCO/VA $_{\text {FEF }_{25 / 75}}$ & 87 & $52-103$ \\
\end{tabular}

$F V C$, Forced vital capacity; $F E V$, forced expiratory volume in 1 second; $D L C O / V A$, diffusing lung capacity corrected for alveolar volume; $F E F_{25 / 75}$, mid-forced expiratory flow.

will be rejected with respect to the HLA combination.

Postoperative pulmonary function testing generally showed a steady improvement that plateaued by postoperative month 9 to 12. For the 15 patients with at least 1 year of follow-up, spirometric results were as follows: mean forced vital capacity was $72 \%$ predicted, mean forced expiratory volume in $1 \mathrm{sec}-$ ond was $73 \%$ predicted, mean mid-forced expiratory flow was $92 \%$, and mean diffusing lung capacity corrected for alveolar volume was $87 \%$ predicted (Table IV).

Because of the obvious concerns regarding whether pulmonary hypertension would develop in two lobes receiving a patient's entire cardiac output, 14 patients who were followed up for at least 1 year underwent right heart catheterization. With the exception of one patient who was in her second trimester of pregnancy and another patient who had unilateral bronchiolitis obliterans with the majority of perfusion going to one lobe, the right heart pressures and pulmonary vascular resistances were within normal ranges. The results for all the patients studied are shown in Table V.

There were two cases of posttransplant lymphoproliferative disease. Both cases responded to a decrease in immunosuppressive therapy as the only therapy.

Bronchiolitis obliterans was definitely diagnosed in three patients. One patient subsequently underwent another bilateral living-donor lobar lung transplant, whereas the other two patients died of their disease. Three additional patients were considered on clinical ground to have early bronchiolitis obliterans manifesting as a decrease in spirometric values. Adjunctive treatment in these six patients included total lymphoid irradiation, methotrexate, photopheresis, antithymocyte $\gamma$-globulin, mycophenolate mofetil, tacrolimus, and cyclophosphamide.

With respect to the lifestyles of the surviving patients, 10 patients have returned to school, seven have returned to work, one is a new mother, and five
Table V. Right heart catheterization results

\begin{tabular}{lcc} 
& Mean & Range \\
\hline RAP (mm Hg) & 3 & $1-8$ \\
PAP (mm Hg) & 20 & $13-38$ \\
PCWP (mm Hg) & 9 & $2-19$ \\
CO (L/min) & 6.3 & $4.7-10.6$ \\
PVR (wood units) & 1.8 & $1.1-4$ \\
\hline
\end{tabular}

$n=14$ patients at 1 year follow-up. $R A P$, Right atrial pressure; $P A P$, pulmonary arterial pressure; $P C W P$, pulmonary capillary wedge pressure; $C O$, cardiac output; $P V R$, pulmonary vascular resistance.

are still within the first 6 postoperative months. All are in New York Heart Association functional class I or II.

Donor outcomes are as important as recipient outcomes in living-donor lobar lung transplantation. Among the 76 donors, complications in the postoperative period included postpericardiotomy syndrome in three patients, atrial fibrillation in one patient, and surgical reexploration in three patients. The diagnoses of the three patients who underwent reexploration were sterile empyema (one), intercostal arterial bleeder (one), and a persistent air leak (one). All of the complications resolved without long-term sequelae. Postoperative spirometric testing of the donors demonstrated a $17 \%$ and $18 \%$ decrease in forced vital capacity and forced expiratory volume in 1 second, respectively. This diminution in pulmonary reserve resulted in no clinical impairment noted by the donors. In a retrospective questionnaire, $96 \%$ of the donors thought of the donation as a positive experience and would be willing to participate again. This survey included donors to recipients who died after operation.

\section{Discussion}

The donor pool is an ever-shrinking resource relative to the growing recipient list. As this discrepancy increases, more and more patients will die while on transplant lists waiting for a suitable cadaveric donor. Living-donor lobar lung transplantation offers a workable alternative to some of these patients when a cadaveric donor cannot be located. This report establishes living-donor lobar lung transplantation as a safe and reproducible procedure, with recipient outcomes comparable to those associated with cadaveric lung transplantation. The outcome data in this report were negatively skewed by severity of illness and the urgent nature of the transplant. Despite this adverse selection, outcomes for these 37 patients were comparable to the national cadaveric lung transplant statistics. 
The 1-year survival of $68 \%$ for patients with cystic fibrosis among our living-donor transplant recipients compares favorably with the national survival of $73 \% .^{9}$

Functional outcome is excellent, as demonstrated by normal hemodynamic and spirometric parameters measured at 1 year. Fourteen patients underwent right heart catheterization, which demonstrated normal right atrial pressures (mean 3.0 $\mathrm{mm} \mathrm{Hg}$ ), normal cardiac output (mean $6.3 \mathrm{~L} / \mathrm{min}$ ), and normal pulmonary vascular resistance (mean 1.8 wood units). These hemodynamic parameters were somewhat surprising, considering that the entire cardiac output is being distributed through two lobes. We believe that this confirms the adaptability of lobar transplantation with time. The spirometric data are equally impressive, with normal forced vital capacity ( $72 \%$ of predicted), forced expiratory volume in 1 second (73\% of predicted), and diffusing lung capacity corrected for alveolar volume (mean $87 \%$ of predicted). The hemodynamic and spirometric data translate clinically to a picture of patients who are active with no restrictions on activities of daily living. Of the 23 surviving patients, 22 are in New York Heart Association functional class $\mathbf{I}$.

The complications noted in this group of patients are comparable to those among patients receiving cadaveric lung transplants. The infection and rejection rates are not different from our cadaveric transplantation. ${ }^{10}$ The pattern of rejection, however, did differ. Of the 30 episodes of rejection seen, 93\% (28) were unilateral; this differs from the cadaveric experience, where rejection is normally bilateral. The rejection severity was less in the living-donor transplant group. Of the 29 episodes of rejection, $96 \%$ were mild and classified as A2 according to the International Society for Heart and Lung Transplantation grading system. The rejection pattern for cadaveric lung transplantation has historically been more severe. These differences in rejection noted are not explained by the HLA matches and mismatches. In a comparison of unilateral versus bilateral rejection, the number of HLA matches and mismatches are seen to be the same in the rejecting and nonrejecting lobes. Obliterative bronchiolitis has remained a problem in livingdonor lobar lung transplantation, as it has been in cadaveric lung transplantation. Three patients (8\%) had tissue confirmation of obliterative bronchiolitis and three additional patients are sus- pected to have obliterative bronchiolitis on the basis of spirometry. In comparison, obliterative bronchiolitis rates in cadaveric lung transplant recipients average between $20 \%$ and $35 \%$, so the combined rate of $16 \%$ (confirmed and suspected) compares favorably. ${ }^{11,12}$

In summary, living-donor lobar lung transplantation is a workable option for patients whose conditions are deteriorating or who are acquiring characteristics that would make them unsuitable candidates for cadaveric lung transplantation. These intermediate data are encouraging with respect to the functional outcome and survival of these critically ill patients, who would have died without this option. The question of whether this operation should be expanded to more elective situations requires more data. Because of the risk to the donor, the weight of the argument still favors living-donor transplantation only for the patient in clinically deteriorating condition. We believe that the data presented here support an expanded role for living-donor lobar lung transplantation.

\section{REFERENCES}

1. Toronto Lung Transplant Group. Experience with single lung transplantation for pulmonary fibrosis. JAMA 1988;259:225862.

2. Egan TM, Kaiser LR, Cooper JD. Lung transplantation. Curr Probl Surg 1989;26:673-752.

3. Starnes VA, Theodore J, Oyer PE, et al. Evaluation of heart-lung transplant recipients with prospective, serial transbronchial biopsies and pulmonary function studies. J Thorac Cardiovasc Surg 1989;98:683-90.

4. Starnes VA, Marshall SE, Lewiston NJ, Theodore J, Stinson EB, Shumway NE. Heart-lung transplantation in infants, children and adolescents. J Pediatr Surg 1991;26:1-4.

5. 1995 Registry, United Network for Organ Sharing. UNOS Update 1996;12:25-7.

6. Starnes VA, Oyer PE, Bernstein D, Baum D, Gamberg P, Miller J, et al. Heart, heart-lung, and lung transplantation in the first year of life. Ann Thorac Surg 1992;53:306-10.

7. Cohen RG, Barr ML, Schenkel FA, DeMeester TR, Wells WJ, Starnes VA. Living-related donor lobectomy for bilateral lobar transplantation in patients with cystic fibrosis. Ann Thorac Surg 1994;57:1423-8.

8. Starnes VA, Barr ML, Cohen RG. Lobar transplantation: indications, technique and outcome. J Thorac Cardiovase Surg 1994;108:403-11.

9. Starnes VA, Lewiston N, Theodore J, Stoehr C, Stinson E, Shumway NE, et al. Cystic fibrosis: target population for lung transplantation in North America in the 1990s. J Thorac Cardiovasc Surg 1992;103:1008-14.

10. Burke CM, Theodore J, Darkins KD, et al. Post-transplant obliterative bronchiolitis and other late lung sequelae in human heart-lung transplantation. Chest 1984;86:824-9.

11. Glanville AR, Baldwin JC, Burke CM, et al. Obliterative bronchiolitis after hear-lung transplantation: apparent arrest 
by augmented immunosuppression. Ann Intern Med 1987; 107:300-4.

12. Theodore J, Starnes VA, Lewiston NJ. Obliterative bronchiolitis. Clin Chest Med 1990;11:309-21.

\section{Discussion}

Dr. Charles B. Huddleston (St. Louis, Mo.). This really is pioneering work, and you should be congratulated for an excellent presentation. In addition, I think that the thoracic surgeons at USC owe you a debt of gratitude for the opportunity of performing operations on 76 healthy, nonsmoking persons without cancer or emphysema.

We have had some experience with this procedure in children at St. Louis, although a much smaller one (just six children), with one death at a mean follow-up of 1 year after transplantation. Our posttransplantation results are fairly similar to yours. We have not had a patient riding a motorcycle, but we did have one on page 2 of the National Enguirer.

One would hope that, as with renal transplants, livingdonor transplants of the lung would in fact have better long-term results than would cadaveric donor transplants. To date, unfortunately, that has not occurred. The pretransplant condition of the recipients, however, may play an important role in this result. Our biggest problem with this procedure is really in deciding how to apply it. At present, our candidates are patients already listed for lung transplantation who are in deteriorating condition and for whom we estimate survival to be only 2 weeks. All six of our recipients have been on the ventilator, paralyzed, with an inspired oxygen fraction of $80 \%$ to $100 \%$ to maintain physiologic blood gas values. Most of them have also required inotropic agents to support their blood pressure. Our reluctance to expand this procedure beyond those who are truly desperately ill is primarily a result of the morbidity imposed on the donors by a thoracotomy and a lobectomy. The somewhat unpredictable nature of cadaveric availability also plays a role in this.

I note that a large portion of your patients were in fact outpatients at the time that they came for their livingdonor lobar lung transplant, and I wonder if you could be more specific about the pretransplant condition of these recipients, particularly those who are outpatients. I think that you answered this in your presentation to some extent already. My next question pertains to the size mismatch. All six of our recipients were children and were much smaller than the donors that were available. The calculated lung volumes that the recipient received actually matched closely with the predicted forced vital capacity and the forced expiratory volume in 1 second for the recipient according to their height. A recent patient whom we evaluated, however, was in fact taller than all of the prospective donors, and we have had some reluctance to transplant to him from living donors because of that. Have you in fact turned down any patients for whom you thought that the prospective donors were too small? Finally, although all of your patients had bilateral transplants, would you consider a single-lobe lung transplant in a patient, appropriately matched with a prospective donor, who has pulmonary fibrosis or pulmonary hypertension and for whom a second prospective donor could not be found?
Dr. Starnes. In looking at the recipients who were outpatients rather than the patients in acute, deteriorating condition, most of these patients were developing panresistant organisms. We know that panresistant infection is a high-risk factor for the patient undergoing conventional cadaveric lung transplantation. I have to say that all patients were reaching a deteriorating status; they were in and out of the hospital for frequent hospitalizations and treatment of their infections. As I pointed out, 25 of the 37 patients were actually in the hospital and in deteriorating condition at the time of transplantation.

With respect to the size mismatch, I think that this is still an inexact science. We transplanted one mismatched case where the recipient was actually 4 to 5 inches taller than the donor. One problem that you get into, as I tried to discuss in the article, is that you have trouble in the acute phase with suction of the chest drains. We found that we cannot initially use any suction. After about 24 hours, you can start applying suction. We found that if we applied suction acutely we could not ventilate the patient. It is almost as though they pull out their lungs to the maximal residual capacity, and you just cannot move the lungs. That is one caveat that we have learned with large size mismatches. Another factor is that the recipient does have drainage for a prolonged period when you have a substantial mismatch. In some of our cases with greater discrepancies, we have had chest drains in for 30 or 35 days, draining 300 to $400 \mathrm{ml} /$ day. The question is whether you can just pull those tubes; as with any lobectomy, or even pneumonectomy, there is obligatory space filling of the area with fluid. Because of the concerns of compression of the recipient lung, however, we have not been willing to do that.

Would I be willing to consider a unilateral lobar transplant? I think the answer is yes. Actually, the first transplant that I did, when I was at Stanford, was a unilateral transplant in a 10 -year-old with pulmonary fibrosis. The donor was the mother, from whom I removed the right upper lobe. I think that if I were doing a unilateral transplant, however, I would probably make sure that the pulmonary artery pressures were not exceptionally high. I think that trying to put all of the cardiac output through one lobe would be a substantial problem.

Dr. Thomas M. Egan (Chapel Hill, N.C.). Dr. Huddleston actually raised two of the issues that I wanted to address. Number one, what is your size limit? I mean, have you ever said to somebody, "No, we cannot do this because you are too big and your donors are too small"? That is a problem that we have run into with adult patients with cystic fibrosis. Second, with respect to the recipients who were outpatients, you mentioned increasing antibiotic resistance as an indication. Have you ever performed a transplant in a patient who had cystic fibrosis and panresistant flora, and have you performed on or offered this operation to patients who are colonized with Burkholderia cepacia?

Dr. Starnes. Taking the last questions together, we have not performed a transplant on a patient with panresistant organisms or with $B$. cepacia, because we think that these factors negatively affect outcomes to a substantial degree. We have turned down patients because we thought that the recipient was just too big for the respective donors. 
We have had some people calling with emphysema, for example, for whom I think that it is inappropriate. We have transplanted in the case of a size discrepancy of 4 to 5 inches in height, knowing that we would have substantial acute and chronic problems with chest drainage, but we have done that only when the patient was truly in a life-threatening situation.

Dr. Otto Gago (Ann Arbor, Mich.). I congratulate you on your outstanding work in this field, but I also pay tribute to Dr. William Adams, who was chairman of the Department of Surgery at the University of Chicago in the early 1960s, and to the group of surgeons doing fundamental work with a similar idea in pulmonary and lobar transplantation. The results of some of that work were presented on several occasions and subsequently published in the Journal (1964;40:726, 1965;50:775, and 1967; 53:109).

I also have a question regarding the indications of patients with pulmonary hypertension. What is your limit with respect to the pulmonary artery pressure and resistance? Also, would you do a unilateral or bilateral lobar lung transplantation, and do you use a cardiopulmonary bypass system in those cases?

Dr. Starnes. We used cardiopulmonary bypass in all of these patients. We believe that it is important to allow reperfusion in a controlled fashion. We actually remove these patients from the heart-lung machine in a slightly hypovolemic state to avoid acute reperfusion of the lungs as much as possible. Most of these patients have acute pulmonary artery pressures of at least a third to even sometimes a half systemic. In terms of whom I would consider for transplantation with pulmonary hypertension, we have done this in two cases. Both patients were children, age 12 and 13 years, and we have only done bilateral lobar transplants. I would not consider a unilateral lobar transplant for a patient with pulmonary hypertension because I believe the pulmonary vascular reserve is inadequate in that situation.

Dr. Michael Bousamra (Milwaukee, Wis.). I congratulate you on your pioneering work, which both offers a viable alternative to patients who are nearing death with end-stage lung disease and also partially alleviates the donor supply problem. I am concerned, however, that the procedure may be being applied too liberally.

I have two questions. During the time that you performed living related-donor bilateral lung transplants for patients with cystic fibrosis, how many cadaveric transplants did you perform? Second, what was the mortality for your patients with cystic fibrosis on the waiting list at that time?

Dr. Starnes. During the concurrent time, we performed 10 cadaveric lung transplants for patients with cystic fibrosis. The death rate of patients on our waiting list has been around $15 \%$ to $20 \%$.

Regarding the overly liberal use of this operation, I think that we have not done that. I think we always take into consideration that we have three patients, two of which are healthy adults, and I believe that the operation is still appropriate for patients who have no other alternative.

\section{Availability of Journal back issues}

As a service to our subscribers, copies of back issues of The Journal of Thoracic and Cardiovascular Surgery for the preceding 5 years are maintained and are available for purchase from Mosby at a cost of $\$ 13.50$ per issue until inventory is depleted. The following quantity discounts are available: $25 \%$ off on quantities of 12 to 23, and one third off on quantities of 24 or more. Please write to Mosby-Year Book, Inc., Subscription Services, 11830 Westline Industrial Drive, St. Louis MO 63146-3318, or call 800-453-4351 or 314-453-4351 for information on availability of particular issues. If unavailable from the publisher, photocopies of complete issues may be purchased from UMI, 300 N. Zeeb Rd., Ann Arbor, MI 48106, 313-761-4700. 